\title{
On the Distribution of Pseudo-Random Numbers Generated by the Linear Congruential Method
}

\author{
By Harald Niederreiter
}

\begin{abstract}
The discrepancy of sequences of pseudo-random numbers generated by the linear congruential method is estimated, thereby improving a result of Jagerman. Applications to numerical integration are mentioned.
\end{abstract}

Let $m$ be a modulus with primitive root $\lambda$, and let $y_{0}$ be an integer in the least residue system modulo $m$ with g.c.d. $\left(y_{0}, m\right)=1$. We generate a sequence $y_{0}, y_{1}, \cdots$ of integers in the least residue system modulo $m$ by $y_{i+1} \equiv \lambda y_{j}(\bmod m)$ for $j \geqq 0$. The sequence $x_{0}, x_{1}, \cdots$, defined by $x_{i}=y_{i} / m$ for $j \geqq 0$, is then a frequently employed sequence of pseudo-random numbers in the unit interval [0,1]. Its elements $x_{i}$ may also be described explicitly by $x_{j}=\left\{\lambda^{j} y_{0} / m\right\}$ for $j \geqq 0$, where $\{x\}$ denotes the fractional part of the real number $x$. The sequence $x_{0}, x_{1}, \cdots$ has period $Q=\phi(m)$, where $\phi$ is Euler's totient function.

For a real number $\alpha$ with $0 \leqq \alpha \leqq 1$, let $A(\alpha)$ be the number of elements of the sequence $x_{0}, x_{1}, \cdots, x_{Q-1}$ lying in the interval $[0, \alpha]$. We define the discrepancy $D=\sup _{0 \leqq \alpha \leqq 1}|A(\alpha) / Q-\alpha|$ which measures the deviation from the uniform distribution. Jagerman [2] has shown that $D \leqq(4 / \pi)((3 \log m) / Q)^{1 / 2}$. His method is based on an estimate of the discrepancy in terms of certain trigonometric sums. In the present note, we shall show that a much simpler method yields a considerably sharper estimate for $D$ (see Theorem 2 ). We prove also some related results.

For $\alpha$ from above and a positive integer $k$, let $A^{(k)}(\alpha)$ be the number of rationals $i / k, 1 \leqq i \leqq k$, g.c.d. $(i, k)=1$, lying in the interval $[0, \alpha]$.

THEOREM 1. For any positive integer $k$, we have

$$
D^{(k)}=\sup _{0 \leqq \alpha \leqq 1}\left|\frac{A^{(k)}(\alpha)}{\phi(k)}-\alpha\right|=O\left(k^{\epsilon-1}\right) \quad \text { for every } \epsilon>0 .
$$

Proof. For an arbitrary positive integer $r$, we consider the sequence of rationals $1 / r, 2 / r, \cdots, r / r$. There are exactly $[r \alpha]$ elements of this sequence in the interval $[0, \alpha]$. We now count these elements by a second method. We write the rationals $j / r$, $1 \leqq j \leqq r$, in reduced form and then count, for each positive divisor $d$ of $r$, the resulting rationals with denominator $d$ lying in $[0, \alpha]$. We thereby arrive at the identity

$$
[r \alpha]=\sum_{d \mid r} A^{(d)}(\alpha) \text { for all } r \geqq 1 \text { and all } \alpha, 0 \leqq \alpha \leqq 1 .
$$

Applying the Moebius inversion formula to (1), we obtain

Received January 6, 1972.

AMS 1970 subject classifications. Primary 65C10, 10F40; Secondary 65D30.

Key words and phrases. Pseudo-random numbers, discrepancy, numerical integration. 


$$
A^{(k)}(\alpha)=\sum_{d \mid k} \mu(d)\left[\frac{k}{d} \alpha\right] \text { for all } k \geqq 1 \text { and all } \alpha, 0 \leqq \alpha \leqq 1 .
$$

Consequently, we have, for all $\alpha$ with $0 \leqq \alpha \leqq 1$,

$$
\begin{aligned}
\left|\frac{A^{(k)}(\alpha)}{\phi(k)}-\alpha\right| & =\left|\frac{1}{\phi(k)} \sum_{d \mid k} \mu(d) \frac{k}{d} \alpha-\frac{1}{\phi(k)} \sum_{d \mid k} \mu(d)\left\{\frac{k}{d} \alpha\right\}-\alpha\right| \\
& =\left|\frac{1}{\phi(k)} \sum_{d \mid k} \mu(d)\left\{\frac{k}{d} \alpha\right\}\right| .
\end{aligned}
$$

Therefore, $D^{(k)} \leqq(1 / \phi(k)) \sum_{d \mid k}|\mu(d)|=g(k)$. Now, $g(k)$ is a multiplicative numbertheoretic function. To prove that $\lim _{k \rightarrow \infty} g(k) k^{1-\epsilon}=0$, it will therefore suffice to show that $\lim _{p^{s \rightarrow \infty}} g\left(p^{s}\right)\left(p^{s}\right)^{1-\epsilon}=0$, where $p^{s}$ runs through all prime powers. But $g\left(p^{s}\right)\left(p^{s}\right)^{1-\epsilon}=2 p^{-\epsilon s}(1-1 / p)^{-1} \leqq 4 p^{-\epsilon s}$, and we are done.

Let us now return to the sequence $x_{0}, x_{1}, \cdots, x_{Q-1}$. Since there is a primitive root modulo $m$, we must have $m=2,4, p^{8}$, or $2 p^{8}$, where $p$ is an odd prime and $s \geqq 1$. For $m=2$ and 4 , we readily get $D=\frac{1}{2}$ and $D=\frac{1}{4}$, respectively. For the remaining cases, we have the following estimates.

THEOREM 2. If $m=p^{s}$, then $D \leqq 1 / Q$. If $m=2 p^{s}$, then $D \leqq 2 / Q$.

Proof. We note that the sequence $x_{0}, x_{1}, \cdots, x_{Q-1}$ runs, in some order, through all the rationals $i / m$ with $1 \leqq i \leqq m$ and g.c.d. $(i, m)=1$. Therefore, $A(\alpha)=A^{(m)}(\alpha)$, and we can apply (2). For $m=p^{s}$, we get, for all $\alpha$ with $0 \leqq \alpha \leqq 1$,

$$
\left|\frac{A(\alpha)}{Q}-\alpha\right|=\frac{1}{Q}\left|\{m \alpha\}-\left\{\frac{m}{p} \alpha\right\}\right|<\frac{1}{Q} \text {. }
$$

For $m=2 p^{s}$, we get, for all $\alpha$ with $0 \leqq \alpha \leqq 1$,

$$
\left|\frac{A(\alpha)}{Q}-\alpha\right|=\frac{1}{Q}\left|\{m \alpha\}-\left\{\frac{m}{2} \alpha\right\}-\left\{\frac{m}{p} \alpha\right\}+\left\{\frac{m}{2 p} \alpha\right\}\right|<\frac{2}{Q} .
$$

It is well known (see for instance [4]) that the discrepancy $D$ of any sequence in $[0,1]$ with $Q$ elements must satisfy $D \geqq 1 / 2 Q$. Therefore, no substantial improvement of Theorem 2 is possible. We refer to [1] for results on the distribution of pseudorandom numbers in the case $m=2^{s}$ with $s \geqq 3$ (of course, $\lambda$ is then not a primitive root any more).

Theorem 2 implies two error estimates for numerical integration based on the sequence $x_{0}, x_{1}, \cdots, x_{Q-1}$. First, we apply Koksma's inequality [3] which states that, for any sequence $a_{0}, a_{1}, \cdots, a_{N-1}$ in $[0,1]$ with discrepancy $D_{N}$ and any integrand $f$ with bounded variation $V(f)$ on $[0,1]$, one has

$$
\left|\frac{1}{N} \sum_{i=0}^{N-1} f\left(a_{i}\right)-\int_{0}^{1} f(x) d x\right| \leqq V(f) D_{N}
$$

The notion of discrepancy is usually defined in terms of the counting functions relative to the half-open intervals $[0, \alpha$, $0<\alpha \leqq 1$. But it is easily seen that this is identical with our concept of discrepancy in which we used the counting functions relative to the closed intervals $[0, \alpha], 0 \leqq \alpha \leqq 1$.

COROLLARY 1. Let $f$ be a function with bounded variation $V(f)$ in $[0,1]$. Then

$$
\left|\frac{1}{Q} \sum_{i=0}^{Q-1} f\left(x_{i}\right)-\int_{0}^{1} f(x) d x\right| \leqq \frac{c}{Q} V(f)
$$

where $c=\frac{1}{2}$ for $m=2$ and $4, c=1$ for $m=p^{s}$, and $c=2$ for $m=2 p^{s}$. 
Finally, we apply an inequality given by the present author in [4]: If $a_{0}, a_{1}, \cdots$, $a_{N-1}$ is a sequence in $[0,1]$ with discrepancy $D_{N}$ and $f$ is continuous in $[0,1]$ with modulus of continuity $\omega$, then

$$
\left|\frac{1}{N} \sum_{i=0}^{N-1} f\left(a_{i}\right)-\int_{0}^{1} f(x) d x\right| \leqq \omega\left(D_{N}\right)
$$

For the convenience of the reader, we include the short proof. We may assume without loss of generality that $0 \leqq a_{0} \leqq a_{1} \leqq \cdots \leqq a_{N-1} \leqq 1$. We know then from [5, Eq. (4)], [6, Theorem 1] that $D_{N}$ is also given by

$$
D_{N}=\max _{i=0, \cdots, N-1} \max \left(\left|a_{i}-\frac{i}{N}\right|,\left|a_{i}-\frac{i+1}{N}\right|\right) .
$$

Now,

$$
\begin{aligned}
\int_{0}^{1} f(x) d x & =\sum_{i=0}^{N-1} \int_{i / N}^{(i+1) / N} f(x) d x \\
& =\sum_{i=0}^{N-1} \frac{1}{N} f\left(\xi_{i}\right) \text { with } \frac{i}{N}<\xi_{i}<\frac{i+1}{N} \text { for } 0 \leqq i \leqq N-1 .
\end{aligned}
$$

Therefore,

$$
\frac{1}{N} \sum_{i=0}^{N-1} f\left(a_{i}\right)-\int_{0}^{1} f(x) d x=\frac{1}{N} \sum_{i=0}^{N-1}\left(f\left(a_{i}\right)-f\left(\xi_{i}\right)\right) .
$$

But $\left|a_{i}-\xi_{i}\right|<\max \left(\left|a_{i}-i / N\right|,\left|a_{i}-(i+1) / N\right|\right) \leqq D_{N}$ for $0 \leqq i \leqq N-1$, hence $\left|f\left(a_{i}\right)-f\left(\xi_{i}\right)\right| \leqq \omega\left(D_{N}\right)$ for $0 \leqq i \leqq N-1$, and we are done.

Using the fact that $\omega$ is a nondecreasing function, we arrive at the following consequence.

COROLlARY 2. Let $f$ be a continuous function in $[0,1]$ with modulus of continuity $\omega$. Then

$$
\left|\frac{1}{Q} \sum_{i=0}^{Q-1} f\left(x_{i}\right)-\int_{0}^{1} f(x) d x\right| \leqq \omega\left(\frac{c}{Q}\right),
$$

where $c$ has the same meaning as in Corollary 1.

Department of Mathematics

University of Illinois at Urbana-Champaign

Urbana, Illinois 61801

1. U. DiETER, "Statistical interdependence of pseudo-random numbers generated by the linear congruential method," Proc. Sympos. on Applications of Number Theory to Numerical Analysis (Montreal, 1971), Academic Press, New York, 1972. (To appear.)

2. D. L. JAGERMAN, "Some theorems concerning pseudo-random numbers," Math. Comp., v. 19,1965 , pp. 418-426. MR 32 \# 1877.

3. J. F. Koxsma, "A general theorem from the theory of uniform distribution modulo 1," Mathematica Zutphen. B, v. 11, 1942, pp. 7-11. (Dutch) MR 7, 370.

4. H. NiederReiter, "Methods for estimating discrepancy," Proc. Sympos. on Applications of Number Theory to Numerical Analysis (Montreal, 1971), Academic Press, New York, 1972. (To appear.)

5. H. NIEDERREITER, "Almost-arithmetic progressions and uniform distribution," Trans. Amer. Math. Soc., v. 161, 1971, pp. 283-292.

6. H. NiederReiter, "Discrepancy and convex programming,"Ann. Mat. Pura Appl., 1972. (To appear.) 